\title{
Metodología para el diseño de intercambiadores de calor tipo coraza y tubos
}

\section{Methodology for the design of shell and tube heat exchangers}

\author{
NUÑEZ-HERNÁNDEZ， Lourdes Nohemi†*'， HORTELANO-CAPETILLO, Juan Gregorio', \\ KANTUN-UICAB, Maria Cristina"“ y TÉLLEZ-MARTÍNEZ, Jorge Sergio”
}

Universidad Politécnica de Juventino Rosas. Academia de Ingeniería Metalúrgica. Hidalgo 102, Comunidad Valenciana, Santa Cruz de Juventino Rosas, Gto, México.

"Universidad Politécnica de Juventino Rosas. Academia de Ingeniería en Plásticos. Hidalgo 102, Comunidad Valenciana, Santa Cruz de Juventino Rosas, Gto, México.

ID $1^{\mathrm{er}}$ Autor: Lourdes Nohemi, Nuñez-Hernández, ORC ID: 0000-0002-3684-7700, CVU CONACYT ID: 1017764

ID $1^{\text {er }}$ Coautor: Juan Gregorio, Hortelano-Capetillo / ORC ID: 0000-0002-3702-4853, CVU CONACYT ID: 347496

ID $2^{\text {do }}$ Coautor: Maria Cristina, Kantun-Uicab / ORC ID: 0000-0003-1588-5414, CVU CONACYT ID: 162342

ID $3^{\text {er }}$ Coautor: Jorge Sergio, Téllez-Martínez / ORC ID: 0000-0003-0587-0059, CVU CONACYT ID: 40084

DOI: $10.35429 / J E A .2020 .23 .7 .7 .18$

Recibido: 15 de Abril, 2020; Aceptado: 29 de Junio, 2020

\section{Resumen}

Los intercambiadores de calor han sido objeto de estudio por más de una centuria. Su clasificación ha abierto diversas ramas de estudio. Dentro de los equipos denominados recuperadores se encuentran los de tipo carcasa y tubos. Estos equipos se utilizan para realizar procesos de refrigeración y calentamiento a partir de circuitos hídricos. Aunque el agua no es la única sustancia de interés, un gran número de trabajos de investigación abordan el comportamiento hidrodinámico acoplado a procesos de intercambio de calor en elementos de diferentes tamaños, con varios arreglos de flujo y de diversos tipos, tal que se desea caracterizarlos en función del desempeño térmico para alcanzar principalmente, alguna temperatura objetivo en los fluidos involucrados, aun cuando esto implique lidiar con grandes caídas de presión. De esta forma, se ha planteado la generación de herramientas computacionales que auxilien a la comprensión de la funcionalidad a ciertas condiciones de operación, así como también la fabricación con certidumbre y bajo costo. Los resultados verificados y validados de una aplicación desarrollada con recursos informáticos accesibles establecen una contribución en este campo activo de la ingeniería.

Intercambiador de calor, procesos térmicos optimizados, simulación de recuperadores, $\varepsilon$-NTU

\begin{abstract}
Heat exchangers have been the subject of study for over a century. Its classification has opened various branches of study. Among the equipment called recuperators are those of the casing and tube type. These equipments are used to carry out cooling and heating processes from water circuits. Although water is not the only substance of interest, a large number of research papers address hydrodynamic behavior coupled to heat exchange processes in elements of different sizes, with various flow arrangements and of various types, such that it is desired to characterize them depending on the thermal performance to mainly achieve some target temperature in the fluids involved, even when this involves dealing with large pressure drops. In this way, the generation of computational tools that help the understanding of functionality under certain operating conditions has been proposed, as well as the manufacturing with certainty and low cost. The verified and validated results of an application developed with accessible computing resources establish a contribution in this active field of engineering.
\end{abstract}

Citación: NUÑEZ-HERNÁNDEZ, Lourdes Nohemi, HORTELANO-CAPETILLO, Juan Gregorio, KANTUN-UICAB, Maria Cristina y TÉLLEZ-MARTÍNEZ, Jorge Sergio. Metodología para el diseño de intercambiadores de calor tipo coraza y tubos. Revista de Aplicaciones de la Ingeniería. 2020. 7-23:7-18.

\footnotetext{
* Correspondencia del Autor (Correo Electrónico: lulisnun@gmail.com)

$\uparrow$ Investigador contribuyendo como primer autor.
} 


\section{Introducción}

Los intercambiadores de calor tipo coraza y tubos son ampliamente usados en la industria gracias a su variedad de construcción y la aplicación que tienen en los procesos de transferencia de calor entre sustancias. La necesidad de la industria por aprovechar la energía ha impulsado tecnologías innovadoras para la fabricación de los intercambiadores de calor bajo las normas de la Asociación de Constructores de Cambiadores de Calor del tipo Tubular (TEMA de sus siglas en inglés). De acuerdo con la clase de intercambiador, estas especifican con detalle: los materiales, las técnicas de diseño, las dimensiones y los métodos de construcción. Lo anterior para determinar el buen funcionamiento mecánico y térmico, es decir, para la optimización de los procesos de transferencia de calor y ahorro de energía. Los intercambiadores de calor pueden clasificarse de acuerdo con criterios tales como la geometría de construcción, los procesos de transferencia y el arreglo de flujos de fluidos, en recuperadores y regenerativos (Kakac \& Hongtan, 1998). De los recuperadores, los más sencillos se pueden definir como cuerpos cilíndricos concéntricos. Estos a su vez se clasifican en: flujo en paralelo o flujo a contracorriente, como se muestra en la Fig. 1 (a) y (b), respectivamente.

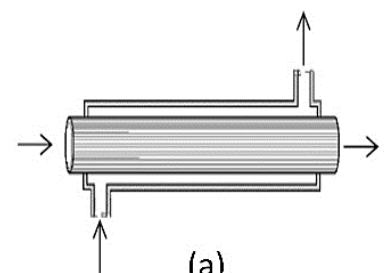

(a)

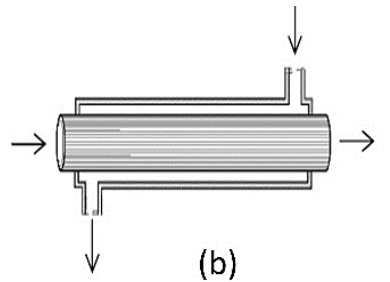

(b)
Figura 1 Intercambiadores de calor concéntricos. Con (a) flujo en paralelo y (b) flujo a contracorriente Fuente: Kakac \& Hongtan, 1998

Otra configuración se estructura con el concepto concéntrico mediante la coraza o carcasa externa y arreglos de tubos. Tales configuraciones se distinguen de acuerdo con la circulación de los fluidos en los tubos y al interior de la carcasa, denominada "paso". En estos intercambiadores normalmente se instalan deflectores (bafles) para aumentar la interacción del fluido al interior de la coraza con los tubos. La Fig. 2 muestra esquemáticamente algunos ejemplos con una configuración geométrica que permite distintos pasos.

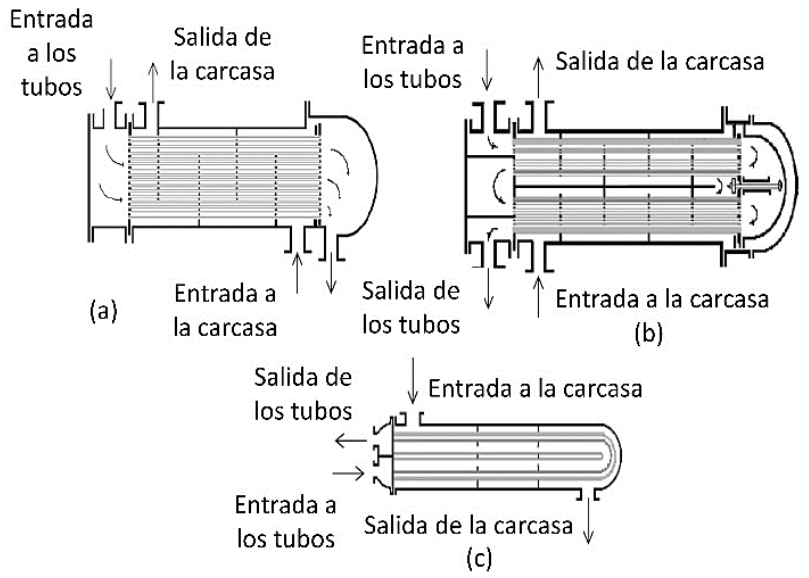

Figura 2 Intercambiadores de Calor Carcasa y Tubos. (a) Un paso por la carcasa y tubos, flujo contracorriente. (b) Dos pasos por la carcasa y cuatro pasos por los tubos. (c) Un paso por la carcasa y dos pasos por los tubos

Fuente: Kakac \& Hongtan, 1998

Considerando la relevancia del control de la transferencia de calor en estos sistemas, en este trabajo se platea una metodología de análisis y se sugiere el uso de una aplicación informática para diseñar un intercambiador de calor tipo carcasa y tubos.

Estos equipos se diseñan con grandes superficies de transmisión de calor asociadas con los diversos tubos montados paralelamente en el interior de la carcasa (Geankoplis, 1993). En relación al tamaño de estos componentes, existen ciertas limitaciones para que los equipos operen tomando en cuenta grandes caídas de presión y su efecto sobre la temperatura de los fluidos (Ozisik, 1990).

Tomando en consideración lo anterior y la información de los estándares de TEMA (Tubular Exchanger Manufacturers Association de sus siglas en inglés) (TEMA, 2007), los esfuerzos para generar metodologías similares a la propuesta, incluyen el análisis de Mehra (1983), quien propone que los estándares de TEMA y ASME se consideren fuentes de información adecuadas para el diseño mecánico y fabricación de los intercambiadores de calor de carcasa y tubos. Con esta referencia, Keene et al. (1994) desarrollaron una aplicación computacional para el cálculo de la temperatura de fluidos para un equipo. Sus resultados fueron validados respecto a los modelos matemáticos propuestos, los cuales integraban los efectos de las caídas de presión del fluido en las secciones delimitadas por los bafles. 
Por otra parte, Yusuf y Ozbilen (2004) realizaron un diseño con predicciones de un código de programación que determina la transferencia de calor óptima en función de los flujos másicos de los fluidos, su temperatura de entrada y salida al proceso, así como el tipo de material de los tubos. La información obtenida específica las dimensiones generales del equipo como son: número de tubos, número de bafles, longitud de los tubos, diámetro interno y externo de los tubos, el área de superficie para la transferencia de calor y las caídas de presión del lado de la carcasa y del lado de los tubos. Asimismo, Leong (1998) desarrolló una aplicación computacional donde diseñan estos equipos alimentando datos de los estándares de TEMA.

La información para el cálculo requiere el conocimiento de: los flujos másicos, la temperatura de entrada y salida de los fluidos del lado de la carcasa y del lado de los tubos, algunas propiedades físicas de fluidos de trabajo como el agua, aire, freon 12, etilenglicol, glicerina y mercurio. En este trabajo se implementa el método de Bell Delaware para determinar los parámetros geométricos del intercambiador de calor (número de tubos, diámetro de la coraza, número de tubos, espaciamiento de bafles, paso pitch, número de pasos en los tubos, etc.). Los investigadores $\mathrm{Su}$, Khin, y Mi (2008) también desarrollaron una aplicación computacional con el objetivo de diseñar intercambiadores como enfriadores de aceite. En su procedimiento intentan obtener la mayor transferencia de energía sin exceder la caída de presión permitida.

Al comparar sus resultados con datos de equipos industriales ajustan sus simulaciones para obtener coeficientes de transferencia de calor internos (del lado tubos) y externos (del lado carcasa) variando el número de bafles, número de tubos y la longitud de los tubos. A su vez, El-Fawal, Fahmy, \& Taher (2011), generaron una aplicación computacional con el objetivo de disminuir al mínimo el costo del diseño al definir la presión optima de los fluidos del lado de la carcasa y del lado tubos. Minimizar el costo implica mejorar el diseño de la geometría del equipo, por lo tanto, para verificar sus resultados realizan una búsqueda de datos en la literatura.
Machuca y Urresta (2008) presentaron la estructura de gráficos y datos determinados por una aplicación informática para el aprendizaje del funcionamiento de los intercambiadores de calor tipo coraza y tubos.

Esta aplicación permite modificar condiciones de diseño y de operación. Similarmente, Tan y Fok (2006) desarrollaron una herramienta informática educativa para el diseño de intercambiadores de calor tipo carcasa y tubos usando el método de Kern para análisis termo hidráulicos. En este programa de cómputo el usuario introduce las especificaciones de diseño y los resultados se muestran dibujos esquemáticos. Los investigadores El-Harbawi, Acellam, \& Yin (2011), presentaron una metodología para desarrollar un programa de cómputo educativo en el lenguaje de programación Visual Basic para diseñar intercambiadores de calor coraza y tubos fundamentado en los métodos de Kern y Bell Delaware.

En el proceso de verificación de resultados se incluyen datos de manuales y de otros programas de diseño. Por su parte, Cartaxo y Fernandes (2010), generaron otro programa en la línea educativa para el diseño de equipos utilizando los métodos de Kern, Bell Delaware y Tinker, proporcionando cursos de operación con ejercicios realistas. Los parámetros que el usuario determina son: los fluidos de trabajo $\left(34^{\circ}\right.$ API, $26^{\circ} \mathrm{API}$ ), la temperatura de entradas en ${ }^{\circ} \mathrm{F}$ $(300,450)$ y salidas en ${ }^{\circ} \mathrm{F}(310,350)$, los flujos másicos en $\operatorname{lbh}^{-1}(72179.79,6900)$ y algunos datos geométricos como diámetros de las tuberías. Otra contribución fue originada por Reyes-Rodríguez et al. (2013), quienes realizaron una modelación térmica e hidráulica de un intercambiador de calor tipo coraza y tubos usando el método $\varepsilon$-NTU y Bell Delaware a través de MATLAB ${ }^{\circledR}$ utilizando, además, una técnica de optimización de algoritmos genéticos para proporcionar un conjunto de Pareto de múltiples soluciones y así minimizar el costo de construcción.

En relación con la mejora de métodos matemáticos, Toledo-Velázquez et al. (2014) presentaron un estudio implementando factores de corrección del método de Bell Delaware para los cálculos de los coeficientes de transferencia de calor y las caídas de presión. 
Aunque el desarrollo se estructuró en FORTRAN 77 se considera una herramienta amigable, en la que el usuario introduce con facilidad parámetros de la geometría del equipo, la temperatura de entrada y las características de los fluidos. Desde el punto de vista físico, Gawande et al. (2012), diseñaron y construyeron un intercambiador de calor coraza y tubos en base a los estándares de TEMA y ASME para fines industriales. Otras referencias de construcción de equipos utilizando herramientas de optimización para disminuir los costos se detallan en las referencias (Reppicha \& Zagermann, 1995), (Babua \& Munawar, 2007), (Fettaka, Thibault, \& Gupta, 2013), (Selbaşa, Kizılkana, \& Reppich, 2006), (Caputo, Pelagagge, \& Salini, 2008), (Ponce-Ortega, Serna-González, \& Jiménez-Gutiérrez, 2009), (Fesangharya, Damangira, \& Soleimani, 2009), (Patel \& Rao, 2010), (Hilbert, Janiga, Baron, \& Thévenin, 2006), (Sanaye \& Hajabdollahi, 2010), (Costa \& Queiroz, 2008), (Rajasekaran \& Kannadasan, 2010).

\section{Metodología}

Contribuyendo al desarrollo de aplicaciones para el diseño de equipos intercambiadores de calor tipo carcasa y tubos, se planteó la generación de una nueva aplicación de cómputo utilizando la interfaz del software ESS (Engineering Equation Solver, de sus siglas en inglés). En el algoritmo de cálculo se establece la solución a la formulación de la transferencia de calor total en el sistema planteando un balance de energía entre los fluidos del lado de la carcasa y del lado de los tubos. La Ec. 1 determina el balance.

$$
Q=\dot{m}_{c a r c} C p_{c a r c}\left(T_{s c}-T_{e c}\right)=m_{\text {tub }} C p_{t u b}\left(T_{e t}-T_{s t}\right)
$$

Donde "m" es representa el flujo másico, "Cp" la capacidad calorífica y "T" valores de temperatura respectivos a los subíndices "carc" que indica la referencia al lado de la carcasa, "ec" a la entrada del lado carcasa, "et" a la entrada del lado tubos, "sc" a la salida del lado carcasa y "st" a la salida del lado tubos.

Debido a que los fluidos que se desplazan a lo largo del intercambiador de calor pierden o adquieren un índice de temperatura local diverso. Una forma de obtener un valor de temperatura representativo se logra mediante la implementación del concepto de la media logarítmica de la diferencia de temperaturas.
La Ec. 2 representa una relación para un flujo de fluido en un sistema a contracorriente. Donde " $\mathrm{T}_{\mathrm{h}}$ " indica la temperatura más alta del fluido $y$ " $T_{c}$ " la temperatura menor. Los índices 1 y 2 representan los datos con referencia al fluido de los lados carcasa y tubos, respectivamente (Kakac \& Hongtan, 1998).

$\Delta T_{m l}=\frac{\left(T_{h 1}-T_{c 2}\right)-\left(T_{h 2}-T_{c 1}\right)}{L n\left[\frac{T_{h 1}-T_{c 2}}{T_{h 2}-T_{c 1}}\right]}$

Puesto que se requiere conocer el área total $\left(\mathrm{A}_{\text {total }}\right)$ asociada a la transferencia de calor; se define la Ec. 3 para aproximar su magnitud como el producto del diámetro externo de los tubos $\left(\mathrm{De}_{\mathrm{tub}}\right)$, su longitud ( $\left.\mathrm{L}_{\mathrm{tub}}\right)$ y el número total de ellos $\left(\mathrm{N}_{\text {tub }}\right)$ (Kakac \& Hongtan, 1998):

$A_{\text {total }}=\pi D e_{t u b} L_{t u b} N_{t u b}$

Lo anterior permite analizar o predecir el rendimiento de un intercambiador de calor, al cuantificar la energía transferida "Q" estimando un coeficiente de transferencia de calor denominado global $\left(\mathrm{U}_{\text {global }}\right)$, tal como lo expresa la Ec. 4 (Kakac \& Hongtan, 1998).

$Q=U_{\text {global }} A_{\text {total }} \Delta T_{m l}$

El cálculo aproximado del coeficiente global de transferencia de calor se obtiene planteando la analogía con un circuito eléctrico en el cual se integran diversas resistencias. Los términos de resistencias asociados a la analogía se expresan como sumandos en el denominador de la Ec. 5. Datos de los coeficientes de transferencia de calor asociados al fluido circulando al interior de los tubos $\left(\mathrm{h}_{\mathrm{i}}\right)$ y en el exterior $\left(h_{0}\right)$, la conductividad en las paredes de los tubos $\left(\mathrm{k}_{\mathrm{mat}}\right)$, su diámetro interno $\left(\mathrm{Di}_{\mathrm{tub}}\right) \mathrm{y}$ externo (De tub , así como, el factor de ensuciamiento $\left(R_{\text {ic }}\right)$, intervienen en la relación.

$$
U_{\text {global }}=\frac{1}{\frac{D e_{t u b}}{D i_{t u b} h_{i}}+\frac{R_{i c} D e_{t u b}}{D i_{t u b}}+\frac{D e_{t u b} \operatorname{Ln}\left(\frac{D e_{t u b}}{D i_{t u b}}\right)}{2 K_{m a t}}+\frac{1}{h_{o}}}
$$

El cálculo de las caídas de presión, a su vez, requiere de la definición del diámetro de la carcasa $\left(D_{\mathrm{s}}\right)$. La Ec. 6 permite establecer una magnitud apropiada en función de las características dimensionales de los tubos:

$D_{S}=0.637 \sqrt{\frac{C L}{C T P}}\left[\frac{A_{t o t a l} P R^{2} D e_{t u b}}{L_{t u b}}\right]^{0.5}$ 
Donde el término CTP adquiere los valores $0.93,0.90$ y 0.85 en relación con si el número de pasos del fluido en el equipo se define como 1, 2 o 3 , respectivamente. Por otra parte, el término CL adquiere el valor de 1.0 o $0.87 \mathrm{si}$, el arreglo de los tubos permite establecer una relación geométrica entre sus centros de, $90^{\circ} \mathrm{y}$ $45^{\circ}$, o de, $30^{\circ}$ y $60^{\circ}$, respectivamente.

De esta forma, la caída de presión en el fluido del lado de los tubos se calcula con la siguiente ecuación (Kakac \& Hongtan, 1998):

$$
\begin{aligned}
& \Delta P_{t}=\left(\frac{4 f L_{t u b} N p}{D i_{t u b}}+4 N p\right)\left(\frac{\rho v^{2}}{2}\right) \\
& f=\left(0.79 \operatorname{Ln}\left(R e_{D}\right)-1.64\right)^{-2}
\end{aligned}
$$

Donde "f" es el factor de fricción estimado a partir del número de Renolds. Las variables " $v$ " y " $\rho$ " representan la velocidad y densidad del fluido, respectivamente. Tals que, la caída de presión en el fluido de lado de la carcasa es:

$$
\begin{aligned}
& \Delta P_{S}=\frac{f G_{S}^{2}(N b+1) D_{S}}{2 \rho D_{e}\left(\frac{\mu}{\mu_{w}}\right)^{0.14}} \\
& f=\exp \left(0.576-0.19 \operatorname{Ln}\left(R e_{S}\right)\right)
\end{aligned}
$$

En resumen, los parámetros geométricos requeridos para especificación son:

- $\quad$ Diámetro interno de los tubos.

- Diámetro externo de los tubos.

- Número de bafles o mamparas.

- $\quad$ Número de tubos.

- Longitud de los tubos.

- $\quad$ Radio Pitch (CL).

- $\quad$ Número de pasos del fluido (CTP).

Por otra parte, se requiere especificar propiedades físicas de los materiales, incluidos los fluidos, tal como:

- Conductividad térmica del material de los tubos.

- Densidad, capacidad térmica y viscosidad de los fluidos.
Adicionalmente, se debe especificar los coeficientes de transferencia de calor. La definición de su magnitud no es arbitraria y por lo tanto, se debe establecer algún mecanismo para utilizar información confiable. El uso de correlaciones para estimar los coeficientes es una estrategia propuesta en este trabajo para concretar el diseño de un equipo intercambiador de calor tipo carcasa y tubos. Las referencias (Colburn, 1993), (Gnielinski, 1976), (Sieder \& Tate, 1936), (Petukhov, 1970), (Sleicher \& Rouse, 1975), así como, (Zukauskas \& Ziugzda, 1985), (Kern, 1965), (Hilpert, 1933), (Bell, 1988), y (Taborek, 1991), se seleccionaron como fuentes confiables de datos de coeficientes de transferencia de calor para caracterizar los fluidos en circulación respecto al interior y el exterior de los tubos.

Correlación de Colburn (Colburn 1993)

$$
N u_{D}=0.023 \operatorname{Re}_{D}^{4 / 5} \operatorname{Pr}^{1 / 3}
$$

Correlación de Gnielinski (Gnielinski 1976)

$$
N u_{D}=\frac{\left(\frac{f}{8}\right)\left(\operatorname{Re}_{D}-1000\right) \operatorname{Pr}}{1+12.7\left(\frac{f}{8}\right)^{0.5}\left(\operatorname{Pr}^{0.66}-1\right)}
$$

Correlación de Sieder-Taste (Sieder 1936)

$$
N u_{D}=0.027 \operatorname{Re}_{D}^{0.8} \operatorname{Pr}^{0.33}\left(\frac{\mu_{b}}{\mu_{w}}\right)^{0.14}
$$

Correlación de Petukov (Petukov 1970)

$$
N u_{D}=\frac{\left(\frac{f}{2}\right) R e_{D} \operatorname{Pr}}{1.07+12.7\left(\frac{f}{2}\right)^{0.5}\left(\operatorname{Pr}^{0.66}-1\right)}
$$

Correlación de Nutter y Sleicher (Sleicher and Rouse 1975)

$$
\begin{gathered}
N u_{D}=5+0.015 R e_{D}^{m} \operatorname{Pr}^{n} \\
m=0.88-\frac{0.24}{4+P r} \\
n=0.33+0.5 \exp (-0.6 P r)
\end{gathered}
$$

Tabla 1 Correlaciones para calcular números de Nusselt asociados al flujo de fluidos al interior de los tubos. Fuente: elaboración propia [MS PowerPoint]

Las Tablas 1 y 2 muestran los datos tabulados de las correlaciones extraídas de las respectivas referencias para la determinación de números adimensionales de Nusselt, de cuya definición, se obtienen los valores de los coeficientes de transferencia de calor necesarios para la simulación; de acuerdo con las características de los procesos planteados en términos de los números adimensionales de Reynolds (Re) y Prandtl (Pr). 
Correlación de Zukauskas (Zukauskas 1987)

$$
\begin{aligned}
& N u_{D}=C R e_{D, \max }^{m} P^{0.36}\left(\frac{P r}{P r_{S}}\right)^{0.25} \\
& 10<\operatorname{Re} \leq 100 \\
& 100<\operatorname{Re} \leq 1000 \\
& 1000<R e \leq 2 E+05
\end{aligned}
$$

\begin{tabular}{cc}
$C$ & $m$ \\
\hline 0.9 & 0.4 \\
0.683 & 0.466 \\
0.35 & 0.6
\end{tabular}

Correlación de Kern (Kern 1950)

$$
\begin{aligned}
& N u_{D}=0.36 \operatorname{Re}_{D}^{0.55} \operatorname{Pr}^{0.33}\left(\frac{\mu}{\mu_{b}}\right)^{0.14} \\
& \text { Para números de Reynolds: }
\end{aligned}
$$

$$
2 \times 10^{3}<R e<1 \times 10^{6}
$$

Correlación de Hilpert (Hilpert 1933)

\begin{tabular}{ccc}
\multicolumn{3}{c}{$N u_{D}=C \operatorname{Re}_{D}^{m} \operatorname{Pr}^{0.33}$} \\
$0.4<\operatorname{Re} \leq 4$ & \multicolumn{1}{c}{$\mathrm{C}$} & $\mathrm{m}$ \\
\cline { 2 - 3 } $4<\operatorname{Re} \leq 40$ & 0.989 & 0.330 \\
$40<\operatorname{Re} \leq 4000$ & 0.683 & 0.385 \\
$4000<\operatorname{Re} \leq 4 E+04$ & 0.193 & 0.466 \\
$4 E+04<\operatorname{Re} \leq 4 E+05$ & 0.027 & 0.618 \\
& & 0.805
\end{tabular}

Correlación de Bell-Delaware (Delaware 1988)

$$
\begin{gathered}
h_{0}=J_{i} C_{p}\left(\frac{\dot{m}}{A_{s}}\right)\left(\frac{k}{C_{p} \mu}\right)^{0.66}\left(\frac{\mu}{\mu_{w}}\right)^{0.14} \\
J_{i}=0.37 R e_{s}^{-0.395}
\end{gathered}
$$

Correlación de Taborek (Taborek 1991)

$$
N u_{D}=0.2 R e_{S}^{0.6} \operatorname{Pr}_{S}^{0.4}
$$

Tabla 2 Correlaciones para calcular números de Nusselt asociados al flujo de fluidos en el exterior de los tubos Fuente: elaboración propia [MS PowerPoint]

La Fig. 3 presenta la interfaz de usuario simplificada y amigable creada para los ejercicios de cálculo que establecen condiciones de diseño de los equipos. Datos relacionados con el flujo volumétrico y la temperatura de entrada de ambos fluidos, así como la presión de alimentación, se definen como condiciones de operación mediante casillas de texto con una etiqueta identificadora.

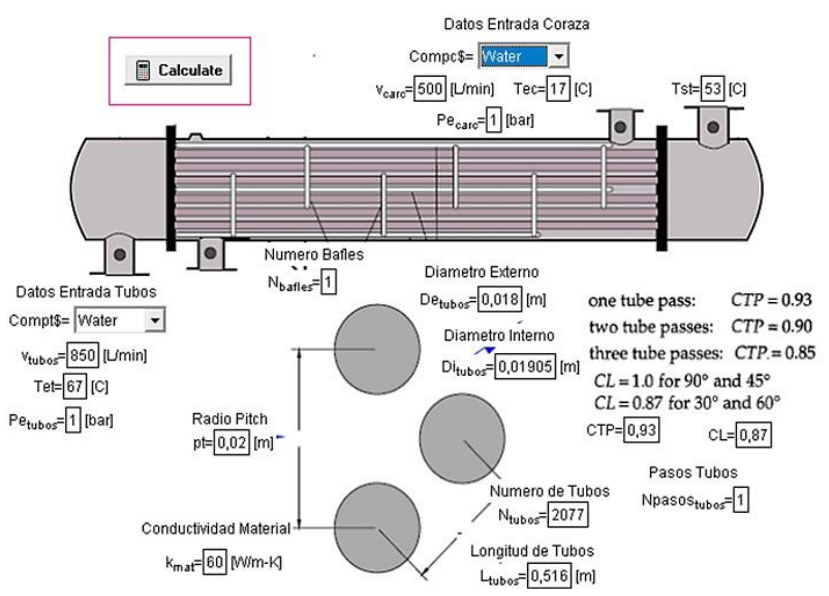

Figura 3 Interfaz de usuario creada en el programa ESS para definir datos respecto al diseño de equipos

Fuente: elaboración propia [Impresión de pantalla interfaz del programa ESS]

ISSN: $2410-3454$

ECORFAN® Todos los derechos reservados
Adicionalmente, la codificación implementa el uso de los métodos del Número de Unidades de Transferencia (NTU de sus siglas en inglés) y el de Número de Eficiencia ( $\varepsilon$ ), ambos números adimensionales dependientes de la capacidad térmica de los fluidos, que permiten obtener una estimación de la temperatura de salida de los fluidos (Reyes-Rodríguez et al., 2013). Para verificar y validar el alcance de los cálculos de la aplicación, se recurrió a información publicada sobre casos teóricos y experimentales, respectivamente.

\section{Resultados}

Se presentan los datos obtenidos de 5 casos de estudio y se analizan con respecto a la predicción del rendimiento térmico e hidráulico de equipos. La Fig. 4 muestra la interfaz de resultados conteniendo datos de una simulación.

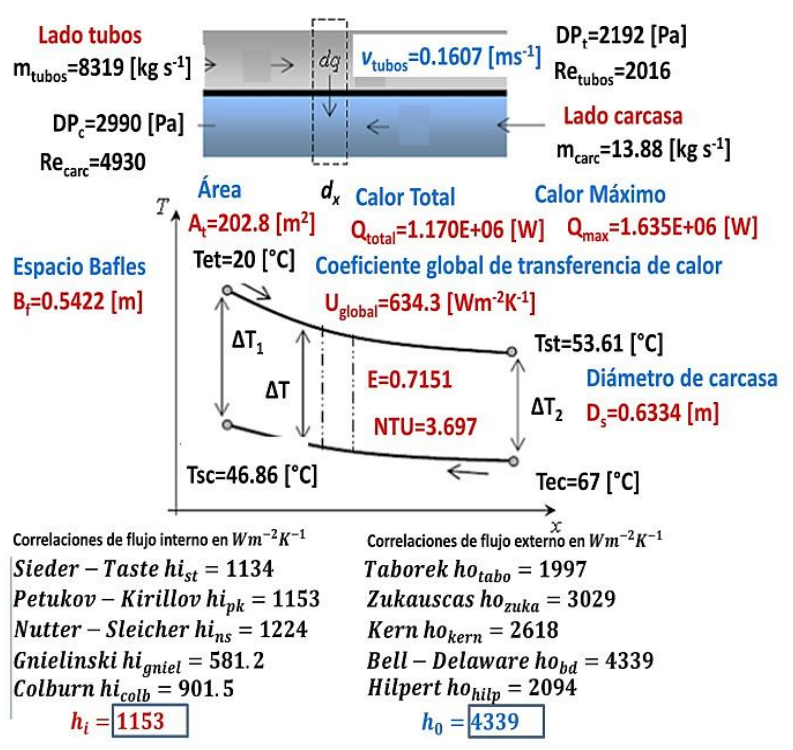

Figura 4 Interfaz de resultados de la simulación presentada al usuario

Fuente: elaboración propia [Impresión de pantalla interfaz del programa ESS]

Como puede notarse, se obtienen resultados de flujos másicos, diámetro de la carcasa, distancia entre bafles, caídas de presión, números de Reynolds, el coeficiente global de transferencia de calor, la temperatura de salida de fluidos, la transferencia de calor total y el área de transferencia de calor. Asimismo, explícitamente se definen coeficientes de transferencia de calor de 5 correlaciones para flujo interno y flujo externo, respectivamente. Dependiendo del tipo de proceso $(\mathrm{Re}, \mathrm{Pr})$, se realizan una serie de combinaciones de las correlaciones para obtener el coeficiente global de transferencia y posteriormente de la temperatura de salida de los fluidos con el método $\varepsilon$-NTU.

NUÑEZ-HERNÁNDEZ, Lourdes Nohemi, HORTELANO-CAPETILLO, Juan Gregorio, KANTUN-UICAB, Maria Cristina y TÉLLEZMARTÍNEZ, Jorge Sergio. Metodología para el diseño de intercambiadores de calor tipo coraza y tubos. Revista de Aplicaciones de la Ingeniería. 2020 


\section{Caso de análisis 1}

La Tabla 3 contiene los datos geométricos y condiciones de operación de un equipo dispuesto como caso de estudio por (Flores-González, 2007). Utilizando la aplicación desarrollada se ejecutaron cuatro simulaciones tomando la información de las condiciones dispuestas al equipo y se cotejaron los resultados obtenidos, en particular, la temperatura de salida de los fluidos.

\begin{tabular}{|c|c|c|}
\hline \multicolumn{3}{|c|}{ Datos } \\
\hline \multicolumn{2}{|c|}{ Diámetro interno tubos } & $14.5 \mathrm{~mm}$ \\
\hline \multicolumn{2}{|c|}{ Diámetro externo de tubos } & $15.5 \mathrm{~mm}$ \\
\hline \multicolumn{2}{|c|}{ Número de tubos } & 55 \\
\hline \multicolumn{2}{|l|}{ Longitud de tubos } & $1163.6 \mathrm{~mm}$ \\
\hline \multicolumn{2}{|l|}{ Paso de Pich } & $19.8 \mathrm{~mm}$ \\
\hline \multicolumn{2}{|l|}{ Número de bafles } & 12 \\
\hline \multicolumn{2}{|l|}{ Pasos en carcasa } & 1 \\
\hline \multicolumn{2}{|l|}{ Pasos en tubos } & 1 \\
\hline \multicolumn{2}{|c|}{ Conductividad del material de tubos } & $55 \mathrm{Wm}^{-2} \mathrm{~K}^{-1}$ \\
\hline \multicolumn{2}{|c|}{ Arreglo de tubos } & Triangular \\
\hline \multicolumn{3}{|c|}{ Caso 1} \\
\hline \multirow{2}{*}{$\begin{array}{l}\text { Fluido: agua } \\
\text { Fujo volumínico, } \mathrm{L} \mathrm{h}^{-1}\end{array}$} & Lado de coraza & Lado de tubos \\
\hline & 14700 & 14700 \\
\hline Flujo másico, $\mathrm{kg} \mathrm{s}^{-1}$ & 4 & 4 \\
\hline \multirow[t]{2}{*}{ Temperatura $\left({ }^{\circ} \mathrm{C}\right)$} & 26.6 & 56.7 \\
\hline & Caso 2 & \\
\hline Fluido: agua & Lado de coraza & Lado de tubos \\
\hline Flujo volumínico, $\mathrm{L} \mathrm{h}^{-1}$ & 13800 & 14700 \\
\hline \multirow{2}{*}{$\begin{array}{l}\text { Flujo másico, } \mathrm{kg} \mathrm{s}^{-1} \\
\text { Temperatura }\left({ }^{\circ} \mathrm{C}\right)\end{array}$} & 3.8 & 4 \\
\hline & 29.8 & 57.3 \\
\hline Temperatura $\left({ }^{\circ} \mathrm{C}\right)$ & Caso 3 & \\
\hline Fluido: agua & Lado de coraza & Lado de tubos \\
\hline \multirow{2}{*}{ Flujo volumínico, $\mathrm{L} \mathrm{h}^{-1}$} & 12900 & 14700 \\
\hline & 3.57 & 4 \\
\hline $\begin{array}{l}\text { Flujo másico, } \mathrm{kg} \mathrm{s}^{-1} \\
\text { Temperatura }\left({ }^{\circ} \mathrm{C}\right)\end{array}$ & 26.7 & 57 \\
\hline \multicolumn{3}{|c|}{ Caso 4} \\
\hline Fluido: agua & Lado de coraza & Lado de tubos \\
\hline \multirow{2}{*}{$\begin{array}{l}\text { Flujo volumínico, } \mathrm{L} \mathrm{h}^{-1} \\
\text { Flujo másico, } \mathrm{kg} \mathrm{s}^{-1}\end{array}$} & 12000 & 14700 \\
\hline & 3.32 & 4 \\
\hline Temperatura $\left({ }^{\circ} \mathrm{C}\right)$ & 26.2 & 56.9 \\
\hline
\end{tabular}

Tabla 3 Datos geométricos y condiciones de proceso de los casos de estudio en el intercambiador de calor utilizado por (Flores-González, 2007)

Fuente: elaboración propia [Ms PowerPoint]

La Tabla 4 contiene la tabulación de la información de ambas fuentes, referencia modelo propuesto, para los cuatro subcasos analizados considerando un equipo con una carcasa de $0.185 \mathrm{~m}$ y $0.1586 \mathrm{~m}$ de diámetro y, para un ajuste de la distancia entre bafles de $0.08325 \mathrm{~m}$ y $0.08951 \mathrm{~m}$, respectivamente. Puede notarse que la temperatura de salida de los fluidos que circulan en el lado carcasa y en al interior de los tubos, difieren en el cálculo con el programa desarrollado entre 1 y $\quad 2 \quad{ }^{\circ} \mathrm{C}$ aproximadamente.
Los coeficientes de transferencia de calor denominados interno y externo que se adoptaron para las simulaciones fueron los respectivos a Nutter_Sleicher y Zukauskas.

\begin{tabular}{|c|c|c|c|}
\hline Dato & Subcaso & $\begin{array}{c}\text { Referencia } \\
{[40]}\end{array}$ & $\begin{array}{c}\text { Modelo } \\
\text { propuesto }\end{array}$ \\
\hline \multirow{4}{*}{$\begin{array}{l}\text { Temperatura } \\
\text { de salida de } \\
\text { coraza. }{ }^{\circ} \mathrm{C}\end{array}$} & 1 & 35.3 & 33.64 \\
\hline & 2 & 37.5 & 36.59 \\
\hline & 3 & 35.6 & 34.56 \\
\hline & 4 & 35.6 & 34.61 \\
\hline \multirow{4}{*}{$\begin{array}{l}\text { Temperatura } \\
\text { de salida de } \\
\text { tubos. }{ }^{\circ} \mathrm{C}\end{array}$} & 1 & 47.9 & 49.58 \\
\hline & 2 & 49.4 & 50.86 \\
\hline & 3 & 48.2 & 50.02 \\
\hline & 4 & 48.9 & 49.95 \\
\hline \multirow{4}{*}{$\begin{array}{l}\text { Transferencia } \\
\text { de calor total. } \\
\text { W }\end{array}$} & 1 & 148313 & 119813 \\
\hline & 2 & 122663 & 108351 \\
\hline & 3 & 132652 & 117389 \\
\hline & 4 & 130341 & 116913 \\
\hline \multirow{4}{*}{ 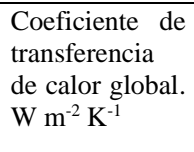 } & 1 & 2224 & 1697 \\
\hline & 2 & 2198 & 1693 \\
\hline & 3 & 2267 & 1676 \\
\hline & 4 & 1976 & 1661 \\
\hline \multirow{4}{*}{$\begin{array}{l}\text { Coeficiente de } \\
\text { transferencia } \\
\text { de calor } \\
\text { interno. W m }{ }^{-2} \\
\mathrm{~K}^{-1}\end{array}$} & 1 & 2763 & 3667 \\
\hline & 2 & 2788 & 3684 \\
\hline & 3 & 2770 & 3676 \\
\hline & 4 & 2777 & 3673 \\
\hline \multirow{4}{*}{$\begin{array}{l}\text { Coeficiente de } \\
\text { transferencia } \\
\text { de calor } \\
\text { externo. } \mathrm{W} \mathrm{m}^{-2} \\
\mathrm{~K}^{-1}\end{array}$} & 1 & 4766 & 9883 \\
\hline & 2 & 4633 & 9628 \\
\hline & 3 & 4427 & 9148 \\
\hline & 4 & 4312 & 8743 \\
\hline \multirow{4}{*}{$\begin{array}{ll}\text { Caída } & \text { de } \\
\text { presión } & \text { en } \\
\text { carcasa. } \mathrm{Pa}\end{array}$} & 1 & 78000 & 35036 \\
\hline & 2 & 68000 & 31018 \\
\hline & 3 & 58000 & 27646 \\
\hline & 4 & 51000 & 24282 \\
\hline \multirow{4}{*}{$\begin{array}{ll}\text { Caída } & \text { de } \\
\text { presión } & \text { en } \\
\text { tubos. Pa } & \end{array}$} & 1 & 7000 & 1332 \\
\hline & 2 & 7000 & 1330 \\
\hline & 3 & 7000 & 1331 \\
\hline & 4 & 7000 & 1331 \\
\hline
\end{tabular}

Tabla 4 Datos de interés para el Caso de análisis 1 tabulados para el cotejo de magnitudes

Fuente: elaboración propia [Ms PowerPoint]

\section{Caso de análisis 2}

La Tabla 5 muestra las condiciones de operación y los parámetros geométricos considerados por Yusuf y Ozbilen (2004), así como, la tabulación de resultados de que permiten encontrar magnitudes similares en la temperatura de salida de fluidos, el diámetro de la carcasa, la distancia entre bafles, el área de transferencia, el coeficiente global y la transferencia de calor total. En la simulación los coeficientes respectivos de transferencia de calor interno y externo fueron los reportados por Nutter_Sleicher y Bell_Delaware. 


\begin{tabular}{|c|c|c|}
\hline \multicolumn{3}{|c|}{ Datos } \\
\hline \multicolumn{2}{|l|}{ Diámetro interno de tubos } & $18 \mathrm{~mm}$ \\
\hline \multicolumn{2}{|l|}{ Diámetro externo de tubos } & $19.05 \mathrm{~mm}$ \\
\hline \multicolumn{2}{|l|}{ Número de tubos } & 2077 \\
\hline \multicolumn{2}{|l|}{ Longitud de tubos } & $516 \mathrm{~mm}$ \\
\hline \multicolumn{2}{|l|}{ Paso de Pitch } & $0.02 \mathrm{~mm}$ \\
\hline \multicolumn{2}{|l|}{ Número de bafles } & 1 \\
\hline \multicolumn{2}{|l|}{ Pasos em carcasa } & 1 \\
\hline \multicolumn{2}{|l|}{ Pasos en tubos } & 1 \\
\hline \multicolumn{2}{|c|}{ Conductividad del material de tubos } & $\begin{array}{r}60 \mathrm{Wm}^{-} \\
{ }^{2} \mathrm{~K}^{-1}\end{array}$ \\
\hline \multicolumn{2}{|l|}{ Arreglo de tubos } & Triangular \\
\hline \multicolumn{3}{|c|}{ Caso 1} \\
\hline Fluido: agua & $\begin{array}{l}\text { Lado de } \\
\text { coraza }\end{array}$ & $\begin{array}{l}\text { Lado de } \\
\text { tubos }\end{array}$ \\
\hline Flujo volumínico, $\mathrm{L} \mathrm{h}^{-1}$ & 500 & 850 \\
\hline Flujo másico, $\mathrm{kg} \mathrm{s}^{-1}$ & 8.33 & 13.88 \\
\hline Temperatura $\left({ }^{\circ} \mathrm{C}\right)$ & 17 & 67 \\
\hline Dato & $\begin{array}{l}\text { Referencia } \\
\text { [7] }\end{array}$ & $\begin{array}{c}\text { Modelo } \\
\text { propuesto }\end{array}$ \\
\hline $\begin{array}{l}\text { Temperatura de salida de } \\
\text { coraza, }{ }^{\circ} \mathrm{C}\end{array}$ & 40 & 39.28 \\
\hline $\begin{array}{l}\text { Temperatura de salida de } \\
\text { tubos, }{ }^{\circ} \mathrm{C}\end{array}$ & 53 & 53.64 \\
\hline Diámetro de coraza & 1.219 & 1.034 \\
\hline Distancia entre bafles, $\mathrm{m}$ & 0.258 & 0.258 \\
\hline Área de transferencia, $\mathrm{m}^{2}$ & 64.15 & 64.14 \\
\hline $\begin{array}{l}\text { Transferencia de calor } \\
\text { total, W }\end{array}$ & 801368 & 775596 \\
\hline $\begin{array}{lr}\begin{array}{l}\text { Coeficiente } \\
\text { transferencia de } \\
\text { global, } \mathrm{Wm}^{-2} \mathrm{~K}^{-1}\end{array} & \begin{array}{r}\text { de } \\
\text { calor }\end{array} \\
\end{array}$ & 422 & 404.1 \\
\hline $\begin{array}{lr}\text { Coeficiente } & \text { de } \\
\text { transferencia de } & \text { calor } \\
\text { interno, } \mathrm{Wm}^{-2} \mathrm{~K}^{-1} & \\
\end{array}$ & - & 490.4 \\
\hline 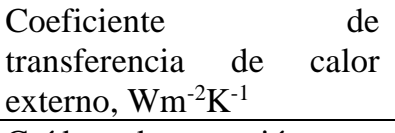 & - & 4431 \\
\hline $\begin{array}{llll}\text { Caída de } & \text { presión } & \text { en } \\
\text { carcasa, } \mathrm{Pa} & & \\
\end{array}$ & 100 & 2123 \\
\hline $\begin{array}{l}\text { Caída de presión en tubos, } \\
\text { Pa }\end{array}$ & 78 & 3.6 \\
\hline
\end{tabular}

Tabla 5 Condiciones de operación, datos geométricos y comparación de resultados del Caso de análisis 2

Fuente: Elaboración propia [Ms PowerPoint]

\section{Caso de análisis 3}

De forma similar, la Tabla 6 contiene los datos de las condiciones de operación y los parámetros geométricos obtenidos del trabajo de Costa y Queiroz, (2008). Introduciendo los parámetros geométricos y las condiciones de operación para la simulación, nuevamente se realizó el proceso de comparación de resultados.

\section{Datos}

Diámetro interno de tubos

Diámetro externo de tubos

Número de tubos

Longitud de tubos

Paso de Pitch

Número de bafles

Pasos em carcasa

Pasos en tubos

Conductividad del material

de tubos

\begin{tabular}{l|l} 
Arreglo de tubos & Triangular
\end{tabular}

\begin{tabular}{|c|c|c|}
\hline \multicolumn{3}{|c|}{ Caso 1} \\
\hline Fluido: agua & $\begin{array}{ll}\text { Lado } & \text { de } \\
\text { coraza } & \end{array}$ & $\begin{array}{l}\text { Lado } \\
\text { tubos }\end{array}$ \\
\hline Flujo volumínico, $\mathrm{L} \mathrm{h}^{-1}$ & 500 & 850 \\
\hline Flujo másico, $\mathrm{kg} \mathrm{s}^{-1}$ & 8.33 & 13.88 \\
\hline Temperatura $\left({ }^{\circ} \mathrm{C}\right)$ & 17 & 67 \\
\hline Dato & $\begin{array}{l}\text { Referencia } \\
\quad[7]\end{array}$ & $\begin{array}{l}\text { Modelo } \\
\text { propuesto }\end{array}$ \\
\hline $\begin{array}{l}\text { Temperatura de salida de } \\
\text { coraza },{ }^{\circ} \mathrm{C}\end{array}$ & 40 & 41.56 \\
\hline $\begin{array}{l}\text { Temperatura de salida de } \\
\text { tubos, }{ }^{\circ} \mathrm{C}\end{array}$ & 53 & 52.27 \\
\hline Diámetro de coraza, $\mathrm{m}$ & 0.438 & 0.4479 \\
\hline Distancia entre bafles, $\mathrm{m}$ & 0.25 & 0.43 \\
\hline Área de transferencia, $\mathrm{m}^{2}$ & 24.63 & 25.84 \\
\hline $\begin{array}{l}\text { Transferencia de calor } \\
\text { total, W }\end{array}$ & 813021 & 855154 \\
\hline $\begin{array}{lr}\begin{array}{l}\text { Coeficiente } \\
\text { transferencia de } \\
\text { global, } \mathrm{Wm}^{-2} \mathrm{~K}^{-1}\end{array} & \begin{array}{r}\text { de } \\
\text { calor }\end{array} \\
\end{array}$ & 1012 & 1184 \\
\hline $\begin{array}{lr}\text { Coeficiente } & \text { de } \\
\text { transferencia de } & \text { calor } \\
\text { interno, } \mathrm{Wm}^{-2} \mathrm{~K}^{-1} & \\
\end{array}$ & - & 6303 \\
\hline $\begin{array}{l}\text { Coeficiente } \\
\text { transferencia de } \\
\text { externo, } \mathrm{Wm}^{-2} \mathrm{~K}^{-1}\end{array}$ & - & 2098 \\
\hline $\begin{array}{lrll}\text { Caída de } & \text { presión } & \text { en } \\
\text { carcasa, } \mathrm{Pa} & & \\
\end{array}$ & 12000 & 20922 \\
\hline $\begin{array}{l}\text { Caída de presión en tubos, } \\
\mathrm{Pa}\end{array}$ & 12000 & 339.4 \\
\hline Velocidad en tubos, $\mathrm{m} \mathrm{s}^{-1}$ & 0.64 & 0.94 \\
\hline
\end{tabular}

Tabla 6 Condiciones de operación, parámetros geométricos y comparación de resultados para el Caso de análisis 3

Fuente: elaboración propia [Ms PowerPoint]

Las temperaturas de salida del lado coraza y tubos tienen un grado de similitud significativo si se utiliza una combinación de los coeficientes de transferencia de calor, interno y externo, correspondientes a las fuentes Nutter_Sleicher y Bell_Delaware, respectivamente.

\section{Caso de análisis 4}

La Tabla 7 muestra información de 3 subcasos de estudio abordados por Leong (1998), cada uno considerando condiciones de operación y datos geométricos de equipo.

NUÑEZ-HERNÁNDEZ, Lourdes Nohemi, HORTELANO-CAPETILLO, Juan Gregorio, KANTUN-UICAB, Maria Cristina y TÉLLEZ MARTÍNEZ, Jorge Sergio. Metodología para el diseño de intercambiadores de calor tipo coraza y tubos. Revista de Aplicaciones de la Ingeniería. 2020 


\begin{tabular}{|c|c|c|c|}
\hline \multicolumn{4}{|c|}{ Subcasos 1, 2,3 } \\
\hline \multicolumn{2}{|l|}{ Estructura } & $\begin{array}{ll}\text { Lado } & \text { de } \\
\text { coraza } & \end{array}$ & $\begin{array}{l}\text { Lado } \\
\text { tubos }\end{array}$ \\
\hline \multicolumn{2}{|c|}{ Fluido } & Aire & Agua \\
\hline \multicolumn{2}{|c|}{ Flujo volumínico, $\mathrm{Lh}^{-1}$} & 11000 & 610 \\
\hline \multicolumn{2}{|c|}{ Flujo másico, $\mathrm{kg} \mathrm{s}^{-1}$} & 1.689 & 10 \\
\hline \multicolumn{2}{|l|}{ Temperatura $\left({ }^{\circ} \mathrm{C}\right)$} & 100 & 30 \\
\hline \multicolumn{2}{|l|}{ Presión, bar } & 10 & 1 \\
\hline Datos & Subcaso 1 & Subcaso 2 & Subcaso 3 \\
\hline $\begin{array}{l}\text { Diámetro } \\
\text { externo } \\
\text { tubos, mm }\end{array}$ & 25 & 25 & 31.7 \\
\hline $\begin{array}{l}\text { Diámetro } \\
\text { externo } \\
\text { tubos, } \mathrm{mm}\end{array}$ & 25.4 & 25.4 & 31.75 \\
\hline $\begin{array}{l}\text { Número } \\
\text { tubos }\end{array}$ & 439 & 497 & 316 \\
\hline $\begin{array}{ll}\text { Longitud } & \mathrm{de} \\
\text { tubos, } \mathrm{mm} & \end{array}$ & 2095 & 19955.8 & 2578.1 \\
\hline $\begin{array}{l}\begin{array}{l}\text { Paso de Pitch, } \\
\mathrm{mm}\end{array} \\
\end{array}$ & 31.75 & 31.75 & 39.69 \\
\hline $\begin{array}{l}\begin{array}{l}\text { Número } \\
\text { bafles }\end{array} \\
\end{array}$ & 3 & 3 & 4 \\
\hline Pasos en carcasa & 1 & 1 & 1 \\
\hline Pasos en tubos & 2 & 2 & 2 \\
\hline $\begin{array}{l}\text { Conductividad } \\
\text { del material de } \\
\text { tubos, } \mathrm{Wm}^{-2} \mathrm{~K}^{-1}\end{array}$ & 60 & 60 & 60 \\
\hline Arreglo de tubos & Cuadrangular & Cuadrangular & Cuadrangular \\
\hline
\end{tabular}

Tabla 7 Condiciones de operación y datos geométricos del Caso de análisis 4

Fuente: elaboración propia [Ms PowerPoint]

En la Tabla 8 puede notarse que en la referencia (Leong, 1998) no presenta datos considerados para la validación. Sin embargo, la temperatura de los fluidos simulada se ajusta relativamente bien, $\left(\begin{array}{llll}3 & \text { a } & 4 & { }^{\circ} \mathrm{C}\end{array}\right)$ cuando se consideran las correlaciones de Gnielinski y Taborek.

\section{Caso de análisis 5}

Finalmente, la Tabla 9 contiene las condiciones de operación y los datos geométricos de los equipos analizado por El-Fawal, Fahmy, \& Taher (2011) y Mizutani et al. (2003). Realizando la comparación del valor de la temperatura de salida de ambos fluidos se observa que la diferencia entre el modelo y las referencias es de alrededor de $5{ }^{\circ} \mathrm{C}$ aproximadamente. Para la simulación se encontró que los datos de los coeficientes de transferencia y calor, interno y externo, a considerar debían ser los definidos por Nutter_Sleicher y Bell_Delaware, respectivamente.

\section{Conclusiones}

El diseño de equipos intercambiadores de calor tipo carcasa y tubos representa una actividad ingenieril que evolutivamente ha adquirido un nivel de sofisticación elevado.

\begin{tabular}{|c|c|c|c|}
\hline Dato & Subcasos & Referencia [8] & $\begin{array}{c}\text { Modelo } \\
\text { propuesto }\end{array}$ \\
\hline \multirow{3}{*}{$\begin{array}{l}\text { Temperatura } \\
\text { de salida de } \\
\text { coraza, }{ }^{\circ} \mathrm{C}\end{array}$} & 1 & \multirow[t]{3}{*}{40} & 37.91 \\
\hline & 2 & & 37.5 \\
\hline & 3 & & 37.66 \\
\hline \multirow{3}{*}{$\begin{array}{l}\text { Temperatura } \\
\text { de salida de } \\
\text { tubos, }{ }^{\circ} \mathrm{C}\end{array}$} & 1 & \multirow[t]{3}{*}{36.1} & 32.54 \\
\hline & 2 & & 32.56 \\
\hline & 3 & & 32.58 \\
\hline \multirow{3}{*}{$\begin{array}{l}\text { Diámetro } \\
\text { coraza, m }\end{array}$} & 1 & 0.838 & 0.783 \\
\hline & 2 & 0.889 & 0.833 \\
\hline & 3 & 0.838 & 0.83 \\
\hline \multirow{3}{*}{$\begin{array}{l}\text { Distancia entre } \\
\text { bafles, } m\end{array}$} & 1 & 0.3352 & 0.52 \\
\hline & 2 & 0.311 & 0.483 \\
\hline & 3 & 0.311 & 0.51 \\
\hline \multirow{3}{*}{$\begin{array}{l}\text { Área de } \\
\text { transferencia, } \\
\mathrm{m}^{2}\end{array}$} & 1 & \multirow[t]{3}{*}{-} & 73.4 \\
\hline & 2 & & 77.56 \\
\hline & 3 & & 81.26 \\
\hline \multirow{3}{*}{$\begin{array}{l}\text { Transferencia } \\
\text { de calor total, } \\
\text { W }\end{array}$} & 1 & \multirow[t]{3}{*}{-} & 107516 \\
\hline & 2 & & 108493 \\
\hline & 3 & & 109341 \\
\hline \multirow{3}{*}{$\begin{array}{l}\text { Coeficiente de } \\
\text { transferencia } \\
\text { de calor global, } \\
\mathrm{Wm}^{-2} \mathrm{~K}^{-1}\end{array}$} & 1 & \multirow[t]{3}{*}{-} & 55.2 \\
\hline & 2 & & 54.25 \\
\hline & 3 & & 51.4 \\
\hline \multirow{3}{*}{$\begin{array}{l}\text { Coeficiente de } \\
\text { transferencia } \\
\text { de } \quad \text { calor } \\
\text { interno, } \mathrm{Wm}^{-} \\
{ }^{2} \mathrm{~K}^{-1}\end{array}$} & 1 & \multirow[t]{3}{*}{-} & 482.6 \\
\hline & 2 & & 408.8 \\
\hline & 3 & & 426.8 \\
\hline \multirow{3}{*}{$\begin{array}{l}\text { Coeficiente de } \\
\text { transferencia } \\
\text { de calor } \\
\text { externo, } \mathrm{Wm}^{-} \\
{ }^{2} \mathrm{~K}^{-1}\end{array}$} & 1 & \multirow[t]{3}{*}{-} & 63.16 \\
\hline & 2 & & 63.42 \\
\hline & 3 & & 59.06 \\
\hline \multirow{3}{*}{$\begin{array}{ll}\text { Caída } & \text { de } \\
\text { presión } & \text { en } \\
\text { carcasa, Pa } & \\
\end{array}$} & 1 & 4430 & 6424 \\
\hline & 2 & 4615 & 6919 \\
\hline & 3 & 4101 & 6815 \\
\hline \multirow{3}{*}{$\begin{array}{ll}\text { Caída } & \text { de } \\
\text { presión } & \text { en } \\
\text { tubos, Pa } & \\
\end{array}$} & 1 & \multirow[t]{3}{*}{-} & 171.7 \\
\hline & 2 & & 131.2 \\
\hline & 3 & & 122.2 \\
\hline \multirow{3}{*}{$\begin{array}{l}\text { Velocidad en } \\
\text { tubos, } \mathrm{m} \mathrm{s}^{-1}\end{array}$} & 1 & \multirow[t]{3}{*}{-} & 0.094 \\
\hline & 2 & & 0.083 \\
\hline & 3 & & 0.081 \\
\hline
\end{tabular}

Tabla 8 Comparación de resultados de los planteamientos del Caso de análisis 4

Fuente: elaboración propia [Ms PowerPoint]

La innovación, sobre todo en la generación de aplicaciones computacionales, adopta las recomendaciones de las investigaciones tanto, para desarrollos teóricos como, de aplicaciones prácticas a nivel laboratorio o industrial. De esta forma, la contribución al desarrollo de estas herramientas, abordando diversos puntos de vista, representa el carácter evolutivo hacia el control deseado para la fabricación de equipos a bajo costo. 


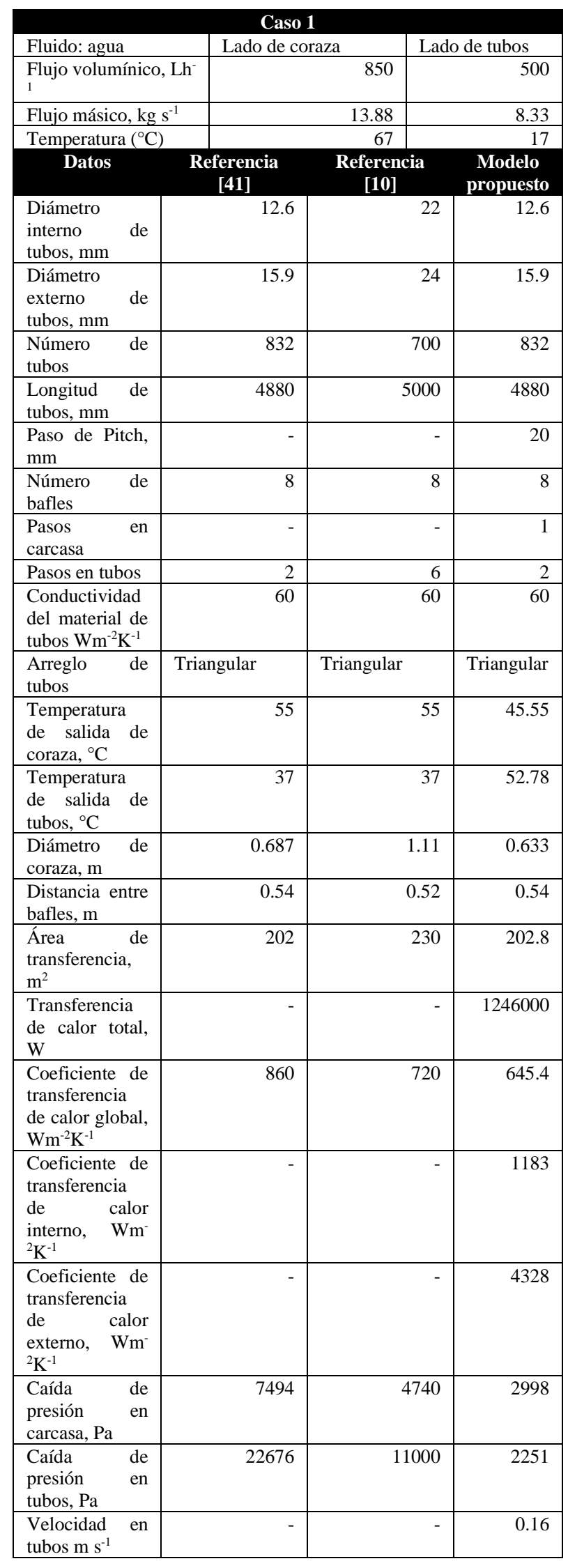

Tabla 9 Comparación de resultados de los planteamientos del Caso de análisis 5 Fuente: elaboración propia [Ms PowerPoint]
En particular, para la aplicación desarrollada en este trabajo, se demuestra que combinando la formulación matemática de la fenomenología implícita en sistemas de flujo a contracorriente y de una herramienta de alto potencial y de fácil accesibilidad como lo es el programa ESS, es posible obtener resultados con elevada rapidez y alta confiabilidad. Puesto que ha sido verificado y validado con información histórica reportada en la literatura en relación a la predicción del rendimiento térmico y mecánico del equipo.

Asimismo, la aplicación provee la información que determina las opciones de diseño y puede utilizarse para ajustar la construcción de equipos a condiciones de operación requeridas, con el análisis de sus posibles variaciones. Lo anterior en principio, para algunas sustancias que son comunes en procesos químicos y que requieren de su procesamiento a ciertas temperaturas específicas.

El uso de correlaciones proporciona una consistencia en la certidumbre para la asignación de coeficientes de transferencia de calor, interno y externo. Para las simulaciones se puede establecer cualquier combinación de éstas que pueda asociarse a un proceso con diferentes condiciones de operación.

\section{Referencias}

Babua, B. V., \& Munawar, S. A. (2007). Differential evolution strategies for optimal design of shell-and-tube heat exchangers. Chemical Engineering Science, 3720-3739.

Bell, K. J. (1988). Heat Transfer Equipment Design. En R. K. Shah, E. Subbarao, \& R. Mashelkar (Ed.), Delaware Method for ShellSide Design (págs. 145-166). New York: Hemisphere Publishing Corporation.

Caputo, A. C., Pelagagge, P. M., \& Salini, P. (2008). Heat exchanger design based on economic optimisation. Applied Thermal Engineering, 1151-1159.

Cartaxo, S. J., \& Fernandes, F. A. (2010). Educational software for heat exchanger equipment. Computer Applications in Engineering Education, 193-199. 
Colburn, A. P. (1993). A method of correlating forced convection heat transfer data and a comparison with fluid friction . Transactions of the American Institute of Chemical Engineers, 174-210.

Costa, A. L., \& Queiroz, E. M. (2008). Design optimization of shell-and-tube heat exchangers. Applied Thermal Engineering, 1798-1805.

El-Fawal, M. M., Fahmy, A. A., \& Taher, B. M. (2011). Modelling of Economical Design of Shell and Tube Type Heat Exchanger Using Specified Pressure Drop. Journal of American Science, 32-40.

El-Harbawi, M., Acellam, D., \& Yin, C. (2011). Development of Educational Software for Designing Shell-and-Tube Heat Exchangers. International Journal of Mechanical Engineering Education, 291-296.

Fesangharya, M., Damangira, E., \& Soleimani, I. (2009). Design optimization of shell and tube heat exchangers using global sensitivity. Applied Thermal Engineering, 1026-1031.

Fettaka, S., Thibault, J., \& Gupta, Y. (2013). Design of shell-and-tube heat exchangers using multiobjective optimization. International Journal of Heat and Mass Transfer, 343-354.

Flores-González, R. (2007). Tesis de Maestría. Fabricación y Validación Experimental de un Intercambiador de Calor de Tubo y Coraza. Ciudad de México, Distrito Federal, México: Instituto Politécnico Nacional.

Gawande, S. H., Wankhede, S. D., Yerrawar, R. N., Sonawane, V. J., \& Ubarhande, U. B. (2012). Design and Development of Shell and Tube Heat Exchanger for Beverage. Modern Mechanical Engineering, 131-125.

Geankoplis, C. J. (1993). Transport Processes and Unit Operations. Englewood Cliffs, New Jersey: Prentice Hall International, Inc.

Gnielinski, V. (1976). New equations for heat and mass transfer in turbulent pipe and channel flow. International Journal of Chemical Engineering, 359-368.
Hilbert, R., Janiga, G., Baron, R., \& Thévenin, D. (2006). Multi-objective shape optimization of a heat exchanger using parallel genetic algorithms. International Journal of Heat and Mass Transfer, 2567-2577.

Hilpert, R. (1933). Wärmeabgabe von geheizten Drähten und Rohren im Luftstrom. Forsch IngWes, 215-224.

Kakac, S., \& Hongtan, L. (1998). Heat Exchangers: Selection, Rating and Thermal Design. Boca Raton, London, New York, Washington D.C.: CRC Press.

Keene, L. W., Gibbons, D. B., Evans, D. J., \& Davies, T. W. (1994). Computer Modeling Validation for Shell and Tube Heat Exchangers. Heat Transfer 1994. Proceedings of the Tenth International Heat Transfer Confrerence (págs. 37-42). Brighton, UK: Chameleon Press Ltd.

Kern, D. Q. (1965). Process Heat Transfer. New York: McGraw-Hill International Book Company.

Leong, Y. C. (1998). Shell and Tube Heat Exchanger Design Software for Educational Applications. International Journal of Engineering Education, 217-224.

Machuca, F., \& Urresta, O. (2008). Software para la enseñanza de la dinámica y control de intercambiadores de calor de tubos y coraza. Revista de la Facultad de Ingeniería de la Universidad de Antioquia , 52-60.

Mehra, D. K. (1983). Shell and Tube Heat Exchangers. Chemical Engineering, 46-56.

Mizutani, F. T., Pessoa, F. L., Queiroz, E. M., Hauan, S., \& Grossmann, I. E. (2003). Mathematical Programming Model for HeatExchanger Network Synthesis Including Detailed Heat-Exchanger Designs. 1. Shell-and-Tube Heat-Exchanger Design. Industrial \& Engineering Chemistry Research , 4009-4018.

Ozisik, M. (1990). Transferencia de calor - Un texto básico. Río de Janeiro: Guanabara Koogan S.A.

Patel, V. K., \& Rao, R. (2010). Design optimization of shell-and-tube heat exchanger using particle swarm optimization technique. Applied Thermal Engineering, 1417-1425. 
Petukhov, B. (1970). Heat Transfer and Friction in Turbulent Pipe Flow with Variable Physical Properties. Advances in Heat Transfer, 503-564.

Ponce-Ortega, J. M., Serna-González, M., \& Jiménez-Gutiérrez, A. (2009). Use of genetic algorithms for the optimal design of shell-andtube heat exchangers. Applied Thermal Engineering, 203-209.

Rajasekaran, S., \& Kannadasan, T. (2010). Optimization of Shell and Tube Heat Exchangers Using modified Genetic Algorithm. International Journal of Control and Automation, 1-10.

Reppicha, M., \& Zagermann, S. (1995). A new design method for segmentally baffled heat exchangers. Computers \& Chemical Engineering, 137-142.

Reyes-Rodríguez, M. B., Moya-Rodríguez, J. L., Cruz-Fonticiella, O., \& Mestizo-Cerón, R. (2013). Diseño de Intercambiadores de Calor de Tubo y Coraza mediante el Método de Eficiencia-Número de Unidades de Transferencia y Optimizacion de los Costos usando MATLAB. Revista Centro Azúcar, 72-80.

Sanaye, S., \& Hajabdollahi, H. (2010). Multiobjective optimization of shell and tube heat exchangers. Applied Thermal Engineering, 19371945.

Selbaşa, R., Kızılkana, Ö., \& Reppich, M. (2006). A new design approach for shell-andtube heat exchangers using genetic algorithms from economic point of view. Chemical Engineering and Processing: Process Intensification, 268-275.

Sieder, E. N., \& Tate, G. E. (1936). Heat Transfer and Pressure Drop of Liquids in Tubes. INDUSTRIAL AND ENGINEERING CHEMISTRY, 1429-1435.

Sleicher, C., \& Rouse, M. (1975). A convenient correlation for heat transfer to constant and variable property fluids in turbulent pipe flow. International Journal of Heat and Mass Transfer, 677-683.

Su, T. M., Khin, A. L., \& Mi, S. M. (2008). Heat Exchanger Design. International Journal of Mechanical, Aerospace, Industrial, Mechatronic and Manufacturing Engineering, 1151-1158.
Taborek, J. (1991). Boilers, Evaporators and Condensers. En S. Kakac (Ed.), Industrial Heat Exchanger Design Practices (págs. 143-176). New York: John Wiley \& Sons, Inc.

Tan, F. L., \& Fok, S. C. (2006). An educational computer-aided tool for heat exchanger design. Computer Applications in Engineering Education, 77-89.

TEMA. (2007). Standard of the Tubular Exchanger Manufacturers Association (Novena ed.). New York: Tubular Exchanger Manufacturers Association, Inc.

Toledo-Velázquez, M., Quinto-Diez, P., Alzelmetti-Zaragoza, J. C., Galvan, S. R., Abugaber-Francis, J., \& Reyes-León, A. (2014). Delaware Method Improvement for the Shell and Tubes Heat Exchanger Design. Engineering, 193-201.

Yusuf, A. K., \& Ozbilen, G. (2004). A Computer Program for Designing of Shell-and-Tube Heat Exchangers. (Elsevier, Ed.) Applied Thermal Engineering, 1797-1805.

Zukauskas, A., \& Ziugzda, J. (1985). Heat transfer of a cylinder in crossflow. New York: Hemisphere Publishing. 